\title{
छs \\ Two dimensional model for coherent synchrotron radiation
}

\author{
Chengkun Huang, Thomas J. T. Kwan, and Bruce E. Carlsten \\ Los Alamos National Laboratory, Los Alamos, New Mexico 87545, USA
}

(Received 19 August 2012; published 10 January 2013)

\begin{abstract}
Understanding coherent synchrotron radiation (CSR) effects in a bunch compressor requires an accurate model accounting for the realistic beam shape and parameters. We extend the well-known 1D CSR analytic model into two dimensions and develop a simple numerical model based on the Liénard-Wiechert formula for the CSR field of a coasting beam. This CSR numerical model includes the 2D spatial dependence of the field in the bending plane and is accurate for arbitrary beam energy. It also removes the singularity in the space charge field calculation present in a 1D model. Good agreement is obtained with 1D CSR analytic result for free electron laser (FEL) related beam parameters but it can also give a more accurate result for low-energy/large spot size beams and off-axis/transient fields. This 2D CSR model can be used for understanding the limitation of various 1D models and for benchmarking fully electromagnetic multidimensional particle-in-cell simulations for self-consistent CSR modeling.
\end{abstract}

DOI: 10.1103/PhysRevSTAB.16.010701

PACS numbers: 41.60.Ap, 41.20.Jb

\section{INTRODUCTION}

Coherent synchrotron radiation is a collective radiation effect in accelerators when high current beam bunches move along a circular beam path, e.g., in a storage ring or in a bunch compressor of a free electron laser (FEL). It is well known that the synchrotron radiation of a single charge particle contains very high harmonics of the rotation frequency. For the radiation wavelength near or longer than the bunched beam size, particles in the beam radiate coherently; therefore the radiation fields are enhanced by the number of coherently emitting particles. These fields can feed back to the beam dynamics, causing emittance growth or microbunching instabilities. Such detrimental effects can degrade the performance of a storage ring or a FEL.

To understand the CSR effects on the beam requires accurate and self-consistent dynamical simulations accounting for the realistic beam shape and parameters, transient dynamics, and possibly a material boundary for CSR suppression. Previous development efforts have led to several multidimensional self-consistent tools for the simulation of CSR effects in realistic devices. For example, Li [1] has developed a Hamiltonian formulation in the internal coordinates of the beam. While in Bassi et al. [2], Gillingham et al. [3], and Novokhatski [4], particle-mesh based methods are used and each adopts a different approach for the solution of the fields: the retarded Green function, the paraxial wave approximation, and an implicit finite-difference solver are employed, respectively. However, it is difficult and time consuming to calculate

Published by the American Physical Society under the terms of the Creative Commons Attribution 3.0 License. Further distribution of this work must maintain attribution to the author(s) and the published article's title, journal citation, and DOI. or simulate CSR self-consistently from first principles therefore idealized theoretical CSR model are often employed in beam tracking codes. Most of the CSR models developed so far use a 1D line charge model [5-8], in which both the beam charge distribution and the field positions are confined to the arc of the circular motion. A single particle wakefield is calculated along the arc using the Liénard-Wiechert formula either for the radiation field only or for a renormalized field which also includes an additional nonsingular Coulomb field component $[5,6]$. The single particle wakefield is then convolved with the smooth 1D line charge density of the beam to obtain the beam wakefield. For highly relativistic beams, the energy dependence of the wakefield is often ignored. Recently, a study of the noise property of the beam on the CSR wakefield suggests that the beam energy has an important role for the level of noise in the CSR field of a realistic beam [9], therefore it is desirable to have accurate energy dependence of the wake. Furthermore, the radiation field exhibit complex distribution in multidimensional space as shown in Sec. IV. While at low beam energy, the space charge force can also affect beam dynamics. In this paper, we extend the well-known 1D CSR analytic model into two dimensions and develop a simple numerical algorithm based on the Liénard-Wiechert formula for the radiation or the total field. This CSR numerical model includes the 2D spatial dependence of the charge and field distributions in the bending plane and can be used for arbitrary beam energy. It also removes the singularity in space charge field calculation and the controversial renormalization process in some of the 1D models. Good agreement is obtained with 1D CSR analytic results for FEL related beam parameters but more accurate results are also found for low-energy or large spot size beams and off-axis fields. This 2D model can be used to understand the limitation of various $1 \mathrm{D}$ models and can be employed to benchmark 
multidimensional fully electromagnetic particle-in-cell (PIC) simulations for self-consistent CSR modeling.

\section{2D CSR MODEL}

In our 2D CSR model, we assume the beam has a distribution in the plane of the reference circular trajectory with a radius $R$ and an origin $O$. We will calculate the free space wakefield of a particle located at its present position $P$ for an observation point $A$ in the same plane. Figure 1 shows the geometric diagram of the $2 \mathrm{D}$ model. Note that, similar to the 1D CSR models, even though we confine source and field points to lower dimensionality in space, the single particle wakefield, i.e., the Green's function, is actually a 3D result evaluated only in the bending plane. The 3D single particle wakefield is discussed in more detail in Ref. [10]; here the 2D model offers a simpler physical picture than a $3 \mathrm{D}$ one while still being a practical method for accurate CSR calculation.

Assuming the emitting particle with charge $e$ moves along the circular trajectory with a constant speed $\beta=$ $|\vec{\beta}|=\left|\vec{\beta}^{\prime}\right|$, one can calculate the retarded position $P^{\prime}$ from which the fields at observation point $A$ and present time $t$ are originated. From the Liénard-Wiechert formula, the electric field at $A$ is

$$
\vec{E}=\frac{e\left(\hat{n}-\vec{\beta}^{\prime}\right)}{\gamma^{2} \rho^{2}\left(1-\hat{n} \cdot \vec{\beta}^{\prime}\right)^{3}}+\frac{e \hat{n} \times\left[\left(\hat{n}-\vec{\beta}^{\prime}\right) \times \dot{\vec{\beta}}^{\prime}\right]}{c \rho\left(1-\hat{n} \cdot \vec{\beta}^{\prime}\right)^{3}},
$$

where $\gamma=\left(1-\beta^{2}\right)^{-1 / 2}$ and $\rho=\overline{P^{\prime} A}$. The first term in Eq. (1) is commonly referred to as the velocity field $\vec{E}^{\mathrm{vel}}$ and the second term as the radiation field $\vec{E}^{\mathrm{rad}}$.

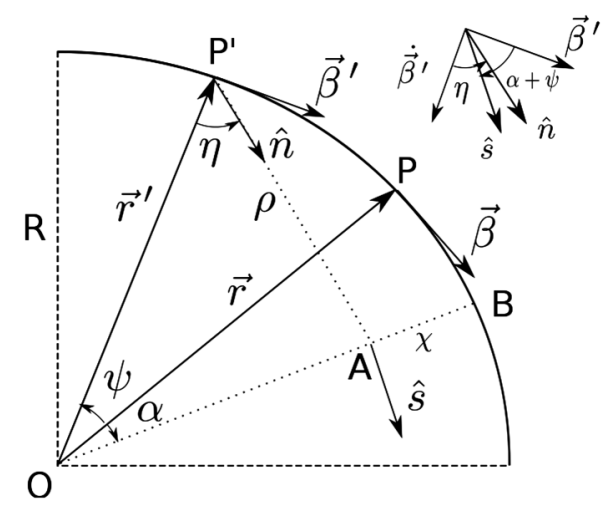

FIG. 1. Diagram of the 2D CSR model. $P$ is the particle's current position on the circular trajectory at time $t, P^{\prime}$ is its position at the retarded time $t^{\prime}$ where the fields at the observation point $A$ is originated from. $\psi$ is the angular separation between $P$ and $P^{\prime}$. The coordinate of $A$ is denoted by its angular separation from $P$ and its radial distance from the trajectory, i.e., $A(\alpha, \chi) . \eta$ is the angle formed between $\overline{P^{\prime} A}$ and $\overline{P^{\prime} O}$. The positive direction of the angles are also shown. $\hat{n}$ and $\hat{s}$ are unit vectors pointing from $P^{\prime}$ to $A$ and perpendicular to $\overline{O A}$, respectively.
The electric field has both transverse and longitudinal components with respect to the velocity of another particle at the observation point $A$. Here we only investigate the longitudinal field. In general, the longitudinal projection of the single particle wakefield not only depends on the bending radius $R$ and the origin $O$ of the emitting particle but also those of the particle at $A$. However, for beam size involved in storage rings and FELs which is typically much smaller than the bending radius $R$, one can ignore these differences and assume that all beam particles move around the same origin with the same angular frequency. Thus, the longitudinal direction is defined here as the tangential direction at point $A$. The longitudinal single particle wakefield weakly depends on $R$ for such small beam sizes. To further simplify the summation of the longitudinal electric field at $A$ from all beam particles, it is assumed that the particle energy is constant and the external magnetic field is uniform. Therefore the wakefield does not depend on the angular position of $P$ and only depends on the relative angular and radial distance between the emitting particle and the observation point. Under these assumptions, the linear superposition of the fields at the observation point can be replaced by a convolution over the beam density profile.

It can be shown that the longitudinal radiation field $E_{s}^{\mathrm{rad}}=\vec{E}^{\mathrm{rad}} \cdot \hat{s}$ is not singular even though $\rho=0$ when $\alpha=\chi=0 . E_{s}^{\mathrm{vel}}=\vec{E}^{\mathrm{vel}} \cdot \hat{s}$ is indeed singular when $\rho=$ 0 . However, unlike the 1D model, this singularity in single particle velocity field does not lead to a diverging result for a smooth beam profile in 2D as we will discuss in Sec. IV and the Appendix. Therefore, it will be included in the calculation in Sec. IV.

\section{RETARDED ANGLE}

For a point particle with charge $e$ moving along a predefined circular trajectory of radius $R$ at a constant speed $\left|\vec{\beta}^{\prime}\right|=|\vec{\beta}|=\beta$ as shown in Fig. 1, we can define the angle between the particle's present position $P$ and the observation point $A$ as $\angle P O A=\alpha$, the retarded angle between $P$ and the retarded position $P^{\prime}$ as $\angle P O P^{\prime}=\psi$ and the angle $\angle O P^{\prime} A=\eta$. In Eq. (1), $\hat{n}, \vec{\beta}^{\prime}, \rho$ all depends on $\psi$ and the field is known to have large spatial variations in some regions; therefore it is important to find an accurate solution for $\psi$ first. In this section, we will use the geometrical relationship illustrated in Fig. 1 to obtain $\psi$ as a function of $\alpha$ and $\chi$. It is generally not possible to obtain an exact answer; therefore various approximate solutions and their accuracy will be discussed in this section.

\section{A. General properties of the retarded angle}

Note that $\widehat{P^{\prime} P}=R \psi=c \beta\left(t-t^{\prime}\right), \overline{P^{\prime} A}=\rho=c\left(t-t^{\prime}\right)$ and $\overline{B A}=\chi$, therefore $\psi / \beta=\rho / R$ and $\overline{O A}=$ $\overline{O B}+\overline{B A}=R+\chi$. From causality, $\psi \geq 0$; we will show later that $\psi=0$ only when $\chi=\alpha=0$. 
For $\triangle A O P^{\prime}$, we have

$$
\begin{aligned}
R^{2}+(R+\chi)^{2}-2 R(R+\chi) \cos (\alpha+\psi) & =\rho^{2}, \\
R^{2}+\rho^{2}-2 R \rho \cos \eta & =(R+\chi)^{2},
\end{aligned}
$$

which can also be rewritten as

$$
\begin{gathered}
1+(1+x)^{2}-2(1+x) \cos (\alpha+\psi)=\psi^{2} / \beta^{2}, \\
1+\psi^{2} / \beta^{2}-2(\psi / \beta) \cos \eta=(1+x)^{2},
\end{gathered}
$$

in which we have adopted the normalized variable $x=\chi / R$.

Equation (2) is valid for both $\alpha \geq 0$ and $\alpha<0$, and from this equation one can solve $\psi$ as a function of $\alpha, x$, and $\beta$; however, this is a transcendental equation which generally requires numerical solution. For theoretical analysis, it is also desirable to obtain a simple approximate solution to Eq. (2) with sufficient accuracy for the parameters of interest. After $\psi$ is solved, one can easily obtain $\eta$ from Eq. (3). Both $\psi$ and $\eta$ are functions of $(\alpha, x, \beta)$ or $(\alpha, x, \gamma)$. Although $\beta$ weakly depends on $\gamma$ for high beam energy, the dependence of $\psi$ on $\gamma$ is not necessarily weak for small $\alpha$ or $x$ as we will discuss below. A plot of $\psi(\alpha)$ for various values of $x$ and $\gamma$ is shown in Fig. 2 for large $\alpha$ and in Fig. 3 for small $\alpha$. It is worth noting that $\psi(\alpha, x, \gamma)$ is periodic in $\alpha$ due to rotation symmetry and it is bounded, i.e., $|x| \leq(\psi / \beta) \leq(2+x)$ from Eq. (2), therefore only the motion of the charge particle during a finite history window will affect the field at a given radius. This result has two practical meanings: (1) a tracking code using the Liénard-Wiechert approach to calculate CSR does not need to store the full trajectory of the particle; (2) once the particle enters the circular trajectory for a sufficient time, the field will reach steady state. For synchrotron radiation calculation at far field, the dependences of $\psi$ on both $\alpha$ and $x$ need to be taken into account as both these arguments are large. While for most of the problems of interest concerning CSR effects on beams, which is the scope of this paper,
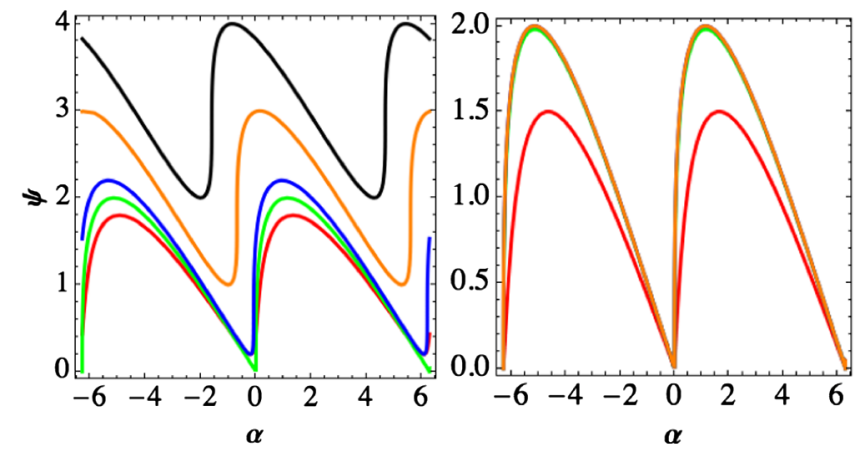

FIG. 2. (left) Retarded angle $\psi$ as a function of $\alpha$ when $\beta=1$, for $x=-0.2,0,0.2,1,2$ (red, green, blue, orange, black curves, respectively). (right) Retarded angle $\psi$ as a function of $\alpha$ when $x=0$, for $\gamma=2,10,100,1000$ (red, green, blue, orange curves, respectively; orange curve is on top of the blue one).
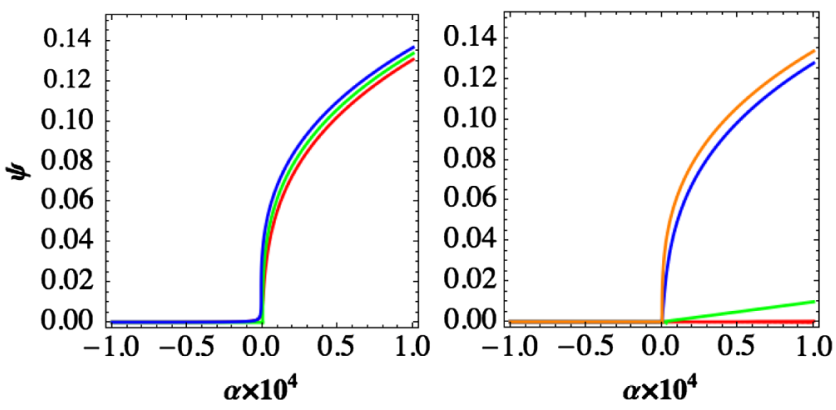

FIG. 3. (left) Retarded angle $\psi$ as a function of $\alpha$ when $\beta=1$ and $\alpha \ll 1$, for $x=-10^{-4}, 0,10^{-4}$ (red, green, blue curves, respectively). (right) Retarded angle $\psi$ as a function of $\alpha$ when $x=0$ and $\alpha \ll 1$, for $\gamma=2,10,100,1000$ (red, green, blue, orange curves, respectively).

$|\alpha|,|x| \ll 1$. In such a case an approximate solution is possible and convenient as discussed by many authors $[5,6,11,12]$.

From Fig. 3, it can be seen that $\psi$ is small when $\alpha \lesssim 0$; therefore the field there is emitted from a retarded position close by. When $\alpha \geq 0, \psi$ quickly increases as $\alpha$ increases, the fields at such an observation point are emitted from a far-away retarded position; the largest value of $\psi$ occurs when both $x$ and $\alpha$ are the largest. A large retarded angle imposes a limit on the phase error of the electromagnetic field in the field solver in a simulation code. For example, to get the electromagnetic field propagation correctly to the observation point at $x=\alpha=10^{-4}$ where $\psi \approx 0.14$ from Fig. 3, the required phase error per wavelength is $\theta_{\max } \lambda /[2 R \sin (\psi / 2)] \approx \theta_{\max } \lambda /(R \psi) \approx 0.0071^{\circ}$, assuming a wavelength of $\lambda / R=2 \times 10^{-4}$ and a maximum accumulated phase error of $\theta_{\max }=5^{\circ}$. Note that at 40 cells per wavelength, the 2 nd order Yee finite-difference time-domain algorithm commonly used in an electromagnetic PIC code has a phase error of about $0.2^{\circ}$ per wavelength; therefore a low dispersion error scheme is necessary for CSR simulation using PIC codes.

\section{B. Approximation for the retarded angle}

A widely used approximation of $\psi$ in 1D models can be obtained from Taylor expansion of the cosine term in Eq. (2) for $x=0$ and $\beta=1$ :

$$
\psi \approx \begin{cases}(24 \alpha)^{1 / 3} & \alpha \geq 0 \\ -\alpha / 2 & \alpha<0\end{cases}
$$

In the above approximation, the first result was obtained by Taylor expansion to the 4th order term and noticing $\psi \gg \alpha$ for $\alpha \geq 0$, hence $\cos (\alpha+\psi) \approx 1-(\alpha+\psi)^{2} / 2+$ $(\alpha+\psi)^{4} / 24 \approx 1-\left(2 \alpha \psi+\psi^{2}\right) / 2+\psi^{4} / 24$. Substituting this into Eq. (2), one finds $\psi=(24 \alpha)^{1 / 3}$. While for the second result, a Taylor expansion to the second order, $\cos (\alpha+\psi) \approx 1-(\alpha+\psi)^{2} / 2$, is sufficient. The comparison of the approximation in Eq. (4) with the exact result is shown in Fig. 4. As expected, Eq. (4) is accurate 


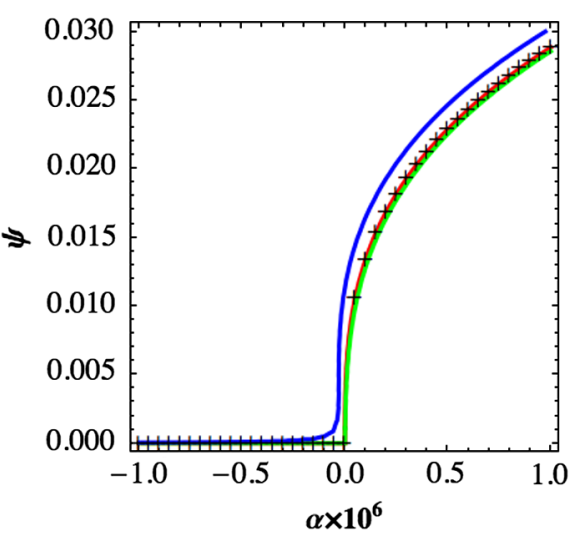

FIG. 4. Retarded angle for small $\alpha$. Red curve is for $x=0$, $\beta=1$; green curve is for $x=0, \gamma=1000$; blue curve is for $x=10^{-5}, \gamma=1000$; black cross is the approximated result from Eq. (4).

for large value of $\gamma$ for $x=0$, but gradually loses accuracy for farther off-axis locations.

However, as shown in Fig. 3, the $\gamma$ dependence of $\psi$ can lead to large error in Eq. (4) at low energy (or small value of $\alpha$ as we will discussed below). References [5,6] use a similar Taylor expansion retaining the energy dependence in Eq. (2). Using $u=\alpha+\psi$, Eq. (4) in Ref. [6] can be rewritten as

$$
(\alpha+\psi)^{3} / 24+(\alpha+\psi)(1-\beta)-\alpha=0,
$$

Similarly, Eq. (3.4) in Ref. [5] is

$$
(\alpha+\psi)^{3} / 24+(\alpha+\psi) /\left(2 \gamma^{2}\right)-\alpha=0 .
$$

Both Eqs. (5) and (6) are only valid for $\alpha>0$. The exact solution of Eq. (6) is

$$
\psi=2\left(\Omega^{1 / 3}-\Omega^{-1 / 3}\right) / \gamma-\alpha,
$$

where

$$
\Omega=3 \gamma^{3} \alpha / 2+\sqrt{\left(3 \gamma^{3} \alpha / 2\right)^{2}+1}
$$

In a 2D model, we need to include the $x$ dependence in the approximate solution of Eq. (2). Here we use the Padé approximant, where the transcendental function $\cos (\zeta)$ is approximated by $P_{l}(\zeta) / Q_{m}(\zeta)$ and $P_{l}, Q_{m}$ are polynomial functions of order $l$ and $m$, respectively. Using $\cos (\zeta) \approx$ $P_{2}(\zeta) / Q_{2}(\zeta)=\left(1-5 \zeta^{2} / 12\right) /\left(1+\zeta^{2} / 12\right)$ and retaining the dependence in $x$ and $\beta$, Eq. (2) can be written as

$$
\begin{aligned}
& \left(x^{2}+\alpha^{2}+x \alpha^{2}+x^{2} \alpha^{2} / 12\right)+\left(2 \alpha+2 x \alpha+x^{2} \alpha / 6\right) \psi \\
& \quad+\left(1+x+x^{2} / 12-1 / \beta^{2}-\alpha^{2} / 12 \beta^{2}\right) \psi^{2} \\
& \quad-\alpha \psi^{3} / 6 \beta^{2}-\psi^{4} / 12 \beta^{2} \approx 0 .
\end{aligned}
$$

Note that for the beam parameters we are mostly interested in, $|x| \ll 1,|\alpha| \ll 1,1 / \beta^{2}-1=\gamma^{-2} \beta^{-2} \ll 1$, and $|2 \alpha \psi| \gg\left|\alpha \psi^{3} / 6 \beta^{2}\right|$, i.e., $12 \beta^{2} \gg \psi^{2}$. Taking $x$ and $\alpha$ to be about the same order of magnitude and keeping
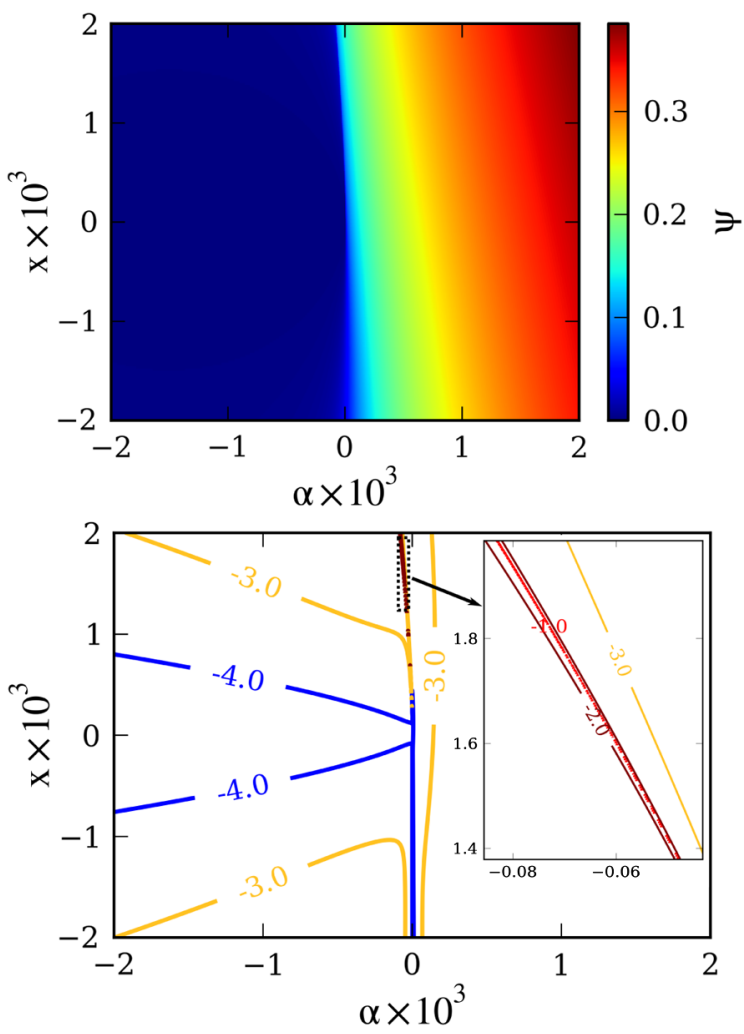

FIG. 5. Top: Retarded angle $\psi_{\text {pade }}$ calculated from Eq. (10) in the region $-2 \times 10^{-3} \leq \alpha, x \leq 2 \times 10^{-3}$ for $R=100 \mathrm{~cm}$, $\gamma=1000$. Bottom: The magnitude of the relative error $\log _{10}\left|\left(\psi_{0}-\psi_{\text {pade }}\right) / \psi_{0}\right|$ for the same range of $\alpha$ and $x$. The inset shows the narrow region where the relative error is largest; the maximum relative error is about $22 \%$.

the leading terms in the coefficients in Eq. (9), then dropping the $\psi^{3}$ term on the left-hand side (LHS) results in

$x^{2}+\alpha^{2}+2 \alpha \psi+\left(x-\gamma^{-2} \beta^{-2}\right) \psi^{2}-\frac{\psi^{4}}{12 \beta^{2}}=0$.

It can be verified that Eq. (10) is consistent with the result in Eq. (4) when $x=0$ and $\beta=1$. In order to check the accuracy of Eq. (10) for off-axis location and finite beam energy, we rely on a numerical solver to find the real and positive root of Eq. (2). Figure 5 shows that the approximate solution using the Padé approximant agrees well with the solution of Eq. (2) for $|x|$ and $|\alpha|$ as large as 0.002 and $\gamma^{-1}$ as large as 0.001 .

\section{Solution for the retarded angle}

Equation (10) has exact analytic solutions but in very complicated form. In order to understand the properties of $\psi$ in the 2D model, we can distinguish several situations, i.e., for small, intermediate, and large value of $\psi$.

Case (1).-A small value of $\psi$, this corresponds to the blue (left) region in Fig. 5. In this case, the $\psi^{4}$ term in Eq. (10) can be dropped as in the 1D model, leading to a 
quadratic equation that can be easily solved with the positive real root being

$\psi_{1}= \begin{cases}\frac{-x^{2}+\alpha^{2}}{2 \alpha} & x=\gamma^{-2} \beta^{-2}, \\ \frac{-\alpha-\sqrt{\alpha^{2}-\left(x^{2}+\alpha^{2}\right)\left(x-\gamma^{-2} \beta^{-2}\right)}}{\left(x-\gamma^{-2} \beta^{-2}\right)} & x \neq \gamma^{-2} \beta^{-2},\end{cases}$

where the second root of the quadratic equation when $x \neq$ $\gamma^{-2} \beta^{-2}$ is dropped due to accuracy consideration discussed below. It can be shown that $\psi_{1}(\alpha<0, x=0) \approx$ $-\alpha / 2$, thus verifying the second result in Eq. (4).

Case (2).-A large value of $\psi$, this corresponds to the red (right) region in Fig. 5. In this case, the $\left(x^{2}+\alpha^{2}\right)$ term in Eq. (10) can be dropped, leading to a cubic equation,

$$
2 \alpha+\left(x-\gamma^{-2} \beta^{-2}\right) \psi-\frac{\psi^{3}}{12 \beta^{2}}=0 .
$$

When $x=0$, we essentially recover Eq. (6). While for $x=\gamma^{-2} \beta^{-2}$, the solution is $\psi_{2}=\left(24 \alpha \beta^{2}\right)^{1 / 3}$, which is consistent with the first result in Eq. (4) albeit with a shift in $x$. For $x \neq \gamma^{-2} \beta^{-2}$, the real root of the cubic equation is

$$
\psi_{2}=4 \beta \Psi \sqrt{\left|x-\gamma^{-2} \beta^{-2}\right|},
$$

where

$$
\Psi= \begin{cases}\sinh \left(\frac{1}{3} \sinh ^{-1} C\right) & x<\gamma^{-2} \beta^{-2} \\ \operatorname{sign}(C) \cosh \left(\frac{1}{3} \cosh ^{-1}|C|\right) & x>\gamma^{-2} \beta^{-2},|C| \geq 1 \\ \cos \left(\frac{1}{3} \cos ^{-1} C\right) & x>\gamma^{-2} \beta^{-2},|C|<1\end{cases}
$$

and $C=1.5 \alpha \beta^{-1}\left|x-\gamma^{-2} \beta^{-2}\right|^{-3 / 2}$.

The above result generally depends on the energy of the particle. However, for $|C| \gg 1$, using the Taylor expansion about infinity, $\sinh ^{-1}(\zeta)=\ln (\zeta)+\ln 2+\zeta^{-2} / 4+\cdots$, and $\sinh (\zeta) \approx e^{\zeta} / 2$, the first result can be approximated as $\Psi \approx(C / 4)^{1 / 3}$, hence $\psi_{2} \approx\left(24 \alpha \beta^{2}\right)^{1 / 3}$ which weakly depends on $\gamma$ and does not depend on $x$. To compare with the result from a 1D model, i.e., $x=0$, we have $C=$ $1.5 \alpha \gamma^{3} \beta^{2}, \quad \psi_{2}=4 \gamma^{-1} \sinh \left[\frac{1}{3} \sinh ^{-1}\left(1.5 \alpha \gamma^{3} \beta^{2}\right)\right]$; when $\alpha \gamma^{3} \gg 1, \psi_{2} \approx\left(24 \alpha \beta^{2}\right)^{1 / 3}$ which is again the familiar result in 1D model in Eq. (4). Therefore, we can conclude that the approximate solution of $\psi$ in Eq. (4) for $\alpha>0$ is accurate when $|C| \gg 1$.

Case (3).-An intermediate value of $\psi$, this corresponds to the middle region in Fig. 5. In this case, one needs to solve the 4th order Eq. (10). However, it is only necessary for a small region in the parameter space; therefore, for convenience we may connect the result in case (1) and case (2) by dropping both $\psi$ and $\psi^{2}$ terms,

$$
\psi_{3}=12 \beta^{1 / 2}\left(x^{2}+\alpha^{2}\right)^{1 / 4},
$$

which is generally a sufficiently good approximation.

Although we have identified three cases that the quartic equation (10) can be further simplified to cubic, quadratic, or even linear equations, the parameter ranges over which we can use these results also need to be discussed. In addition to the requirement that $\psi$ is a real positive root of these equations, the relative error with respect to the true root $\psi_{0}$ of Eq. (10) needs to be small. The relative error can be estimated by the Taylor expansion of the LHS of Eq. (10) $g(\psi) \equiv x^{2}+\alpha^{2}+2 \alpha \psi+\left(x-\gamma^{-2} \beta^{-2}\right) \psi^{2}-$ $\psi^{4} /\left(12 \beta^{2}\right)$. For example, expanding $g\left(\psi_{0}\right)$ around the root $\psi_{1}$ of the quadratic equation,

$$
g\left(\psi_{0}\right)=0 \approx g\left(\psi_{1}\right)+g^{\prime}\left(\psi_{1}\right)\left(\psi_{0}-\psi_{1}\right),
$$

where $g^{\prime}(\psi)=d g(\psi) / d \psi=2 \alpha+2\left(x-\gamma^{-2} \beta^{-2}\right) \psi-\psi^{3} /$ $\left(4 \beta^{2}\right)$, one obtains the relative error $\epsilon \equiv\left|\left(\psi_{0}-\psi_{1}\right) / \psi_{1}\right|$,

$$
\begin{aligned}
\epsilon & =\left|\frac{g\left(\psi_{1}\right)}{\psi_{1} g^{\prime}\left(\psi_{1}\right)}\right| \\
& =\left|\frac{\psi_{1}^{3}}{12 \beta^{2}\left[2 \alpha+2\left(x-\gamma^{-2} \beta^{-2}\right) \psi_{1}-4 \psi_{1}^{3}\right]}\right| .
\end{aligned}
$$

Substituting the solution $\psi_{1}$, and enforcing the requirement $\epsilon \ll 1$ and $\psi_{1}$ to be a real positive number, it is found that $\psi_{1}$ is a good approximation when $\alpha<0$ and $\alpha^{2} \gg$ $\frac{3}{2}\left(x-\gamma^{-2} \beta^{-2}\right)^{2}-x^{2}$. A similar procedure can be used to determine the error of the approximated result in the other cases without numerically solving Eq. (10).

\section{LONGITUDINAL SINGLE PARTICLE WAKEFIELD}

With $\psi$ and $\eta$ solved using the numerical or approximate methods described in Sec. III, Eq. (1) can be used to calculate the free space wakefield of a single particle in circular motion. The following identities are useful in the expression of the electric field:

$\hat{n} \cdot \vec{\beta}^{\prime}=\beta \cos (\pi / 2-\eta)=\beta \sin \eta$,

$\hat{n} \cdot \dot{\vec{\beta}}^{\prime}=\frac{\beta^{2} c}{R} \cos \eta$,

$\hat{n} \cdot \hat{s}=\cos (\eta+\alpha+\psi-\pi / 2)=\sin (\eta+\alpha+\psi)$,

$\vec{\beta}^{\prime} \cdot \hat{s}=\beta \cos (\alpha+\psi), \quad \dot{\vec{\beta}}^{\prime} \cdot \hat{s}=\frac{\beta^{2} c}{R} \sin (\alpha+\psi)$.

For the radiation field, the tangential component $E_{s}^{\mathrm{rad}}=\vec{E}^{\mathrm{rad}} \cdot \hat{s}$ is

$$
E_{s}^{\mathrm{rad}}=\frac{e\left[\left(\hat{n} \cdot \dot{\vec{\beta}}^{\prime}\right)\left(\hat{n} \cdot \hat{s}-\vec{\beta}^{\prime} \cdot \hat{s}\right)-\left(\hat{s} \cdot \dot{\vec{\beta}}^{\prime}\right)\left(1-\hat{n} \cdot \vec{\beta}^{\prime}\right)\right]}{c \rho\left(1-\hat{n} \cdot \vec{\beta}^{\prime}\right)^{3}},
$$

while for the velocity field

$$
E_{s}^{\mathrm{vel}}=\frac{e\left(\hat{n} \cdot \hat{s}-\vec{\beta}^{\prime} \cdot \hat{s}\right)}{\gamma^{2} \rho^{2}\left(1-\hat{n} \cdot \vec{\beta}^{\prime}\right)^{3}}
$$


Using the identities above and $\rho=\psi R / \beta$, we obtain

$$
E_{s}^{\mathrm{rad}}=\frac{e \beta^{3}(\sin \eta-\beta) \cos (\eta+\alpha+\psi)}{R^{2} \psi(1-\beta \sin \eta)^{3}},
$$

and

$$
E_{s}^{\mathrm{vel}}=\frac{e \beta^{2}[\sin (\eta+\alpha+\psi)-\beta \cos (\alpha+\psi)]}{\gamma^{2} R^{2} \psi^{2}(1-\beta \sin \eta)^{3}} .
$$

It is also useful to obtain $E_{s}$ through the scalar and vector potentials,

$$
E_{s}=\vec{E} \cdot \hat{s}=-\frac{\partial \phi}{\partial s}-\frac{\partial A_{s}}{c \partial t}=-\frac{\partial\left(\phi-\beta A_{s}\right)}{\partial \xi},
$$

where $\phi, A_{s}=\vec{A} \cdot \hat{s}$ are the scalar potential and the longitudinal component of the vector potential in the Lorentz gauge, respectively, and $\xi=s-c \beta t=\alpha R . \phi, \vec{A}$ are also given by the Liénard-Wiechert formula, therefore

$$
\left(\phi-\beta A_{s}\right)=\frac{e\left(1-\beta \vec{\beta}^{\prime} \cdot \hat{s}\right)}{\rho\left(1-\hat{n} \cdot \vec{\beta}^{\prime}\right)}=\frac{e \beta\left[1-\beta^{2} \cos (\alpha+\psi)\right]}{R \psi(1-\beta \sin \eta)} .
$$

\section{A. On-axis single particle wakefield}

When the observation position $A$ is on the same trajectory of the charge, i.e., $x=0$, the above expressions are much simplified by noticing that $\sin ^{2}\left(\frac{\alpha+\psi}{2}\right)=\psi^{2} / 4 \beta^{2}$ and $\psi \cos \eta=\psi^{2} / 2 \beta$ from Eqs. (2) and (3). Consider $\psi=0$; it is obvious that $\alpha=\psi=0$, also $\eta$ is not relevant and can be set to 0 too. If $\alpha>0, \sin \left(\frac{\alpha+\psi}{2}\right)=\cos \eta=\psi / 2 \beta$, while for $\alpha<0$, since $\beta=\left|2 \sin \left(\frac{\alpha+\psi}{2}\right) / \psi\right| \leq 1, \quad \sin \left(\frac{\alpha+\psi}{2}\right)=$ $-\cos \eta=-\psi / 2 \beta$. Therefore, one can write

$$
\alpha+\psi+2 \eta= \begin{cases}\pi & \alpha>0 \\ 0 & \alpha=0 \\ -\pi & \alpha<0\end{cases}
$$

and

$$
E_{s}^{\mathrm{rad}}(x=0)= \begin{cases}\frac{e \beta^{2}(\beta-\sin \eta)}{2 R^{2}(1-\beta \sin \eta)^{3}} & \alpha \neq 0 \\ -\frac{e \beta^{2}}{2 R^{2}(1-\beta)^{2}} & \alpha \rightarrow 0^{+} \\ \frac{e \beta^{2}}{2 R^{2}(1+\beta)^{2}} & \alpha \rightarrow 0^{-} \\ \frac{e \beta^{2}}{4 R^{2}}\left[\frac{1}{(1+\beta)^{2}}-\frac{1}{(1-\beta)^{2}}\right] & \alpha=0 .\end{cases}
$$

Note that $E_{s}^{\mathrm{rad}}(x=0)$ is not singular for any $\alpha$ but it is discontinuous at $\alpha=0$. For $\gamma \gg 1, E_{s}^{\mathrm{rad}}\left(\alpha \rightarrow 0^{+}\right.$, $x=0) \approx-2 e \beta^{2} \gamma^{4} / R^{2} \quad$ while $\quad E_{s}^{\mathrm{rad}}\left(\alpha \rightarrow 0^{-}, x=0\right) \approx$ $e /\left(8 R^{2}\right), \quad E_{s}^{\mathrm{rad}}\left(\alpha \rightarrow 0^{+}, x=0\right) / E_{s}^{\mathrm{rad}}\left(\alpha \rightarrow 0^{-}, x=0\right)=$ $-(1+\beta)^{2} /(1-\beta)^{2} \approx-16 \gamma^{4}$. The value of $E_{s}^{\text {rad }}$ at this point is conveniently defined as the average of $E_{s}^{\mathrm{rad}}\left(\alpha \rightarrow 0^{+}, x=0\right)$ and $E_{s}^{\mathrm{rad}}\left(\alpha \rightarrow 0^{-}, x=0\right)$. Although not rigorous, this definition is consistent with the

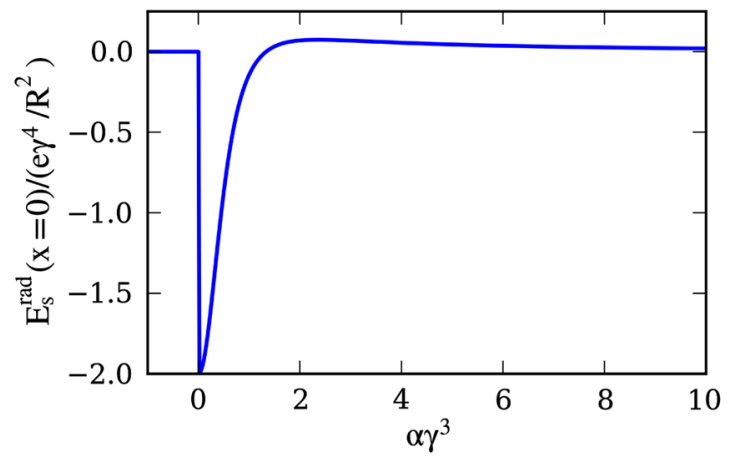

FIG. 6. On-axis radiation wakefield $E_{s}^{\mathrm{rad}}(x=0)$ for a single particle.

instantaneous power loss of the emitting electron from the Larmor formula in the point charge model [5]. Using the solution of Eq. (6), $E_{s}^{\text {rad }}(x=0)$ can be completely determined. $E_{s}^{\text {rad }}(x=0)$ is well known in the 1D models for its angular-energy scaling parameter $\alpha \gamma^{3}$ which can be seen from Eq. (8). $E_{s}^{\mathrm{rad}}(x=0)$ is plotted in Fig. 6, which consists of a negligible part for $\alpha<0$, a pronounced negative peak for $0<\alpha \gamma^{3} \leqslant 1$, and a far field that drops off slowly as $\alpha^{-4 / 3}$ for $\alpha \gamma^{3} \gg 1$. It is the far field that leads to the characteristic parabolic cylinder function shape of the CSR field for a smooth Gaussian beam. However, the spiky feature in $E_{s}^{\mathrm{rad}}(x=0)$ can also play an important role if the beam current has modulation with scale length on the order of $\gamma^{-3} R$ which is in resonance with the negative peak. Because the synchrotron radiation cone has a finite angular extent, it is not surprising that the negative peak also has an extent in the radial direction in a 2D model as discussed below.

Similarly, we can obtain the expression of $\left(\phi-\beta A_{s}\right)$ for $x=0$ from Eq. (17). For small $\alpha$ and $\beta=1$, $\left(\phi-\beta A_{s}\right)$ can be approximated using Eq. (4). After some algebra, it can be written as

$$
\left(\phi-A_{s}\right) /(e / R) \approx \begin{cases}2 /(3 \alpha)^{1 / 3} & \alpha \geq 0 \\ -\alpha / 8 & \alpha<0\end{cases}
$$

As discussed in Sec. III, the first result in the above approximation is valid for $\alpha \gg \gamma^{-3}$.

\section{B. Off-axis single particle wakefield}

For the off-axis locations, one can show that $E_{s}^{\mathrm{rad}}(x \neq 0)$ is not singular either; this is because $(1-\beta \sin \eta)>0$ and $\psi(x \neq 0)>0$. The latter inequality is valid because $\psi=$ 0 implies that $x=0$ in Eq. (3). $E_{s}^{\text {rad }}$ from Eq. (14), which is shown in Fig. 7, exhibits a complicated pattern in two dimensional $(\alpha, x)$ space. Similar to the 1D result, for a high energy particle, $E_{s}^{\text {rad }}$ only depends on $\alpha$ through the quantity $\alpha \gamma^{3}$, while the $x$ dependence is through the quantity $x \gamma^{2}$. This can be understood by noting that Eq. (10) can be rewritten, by ignoring the second term $\alpha^{2}$ and setting 


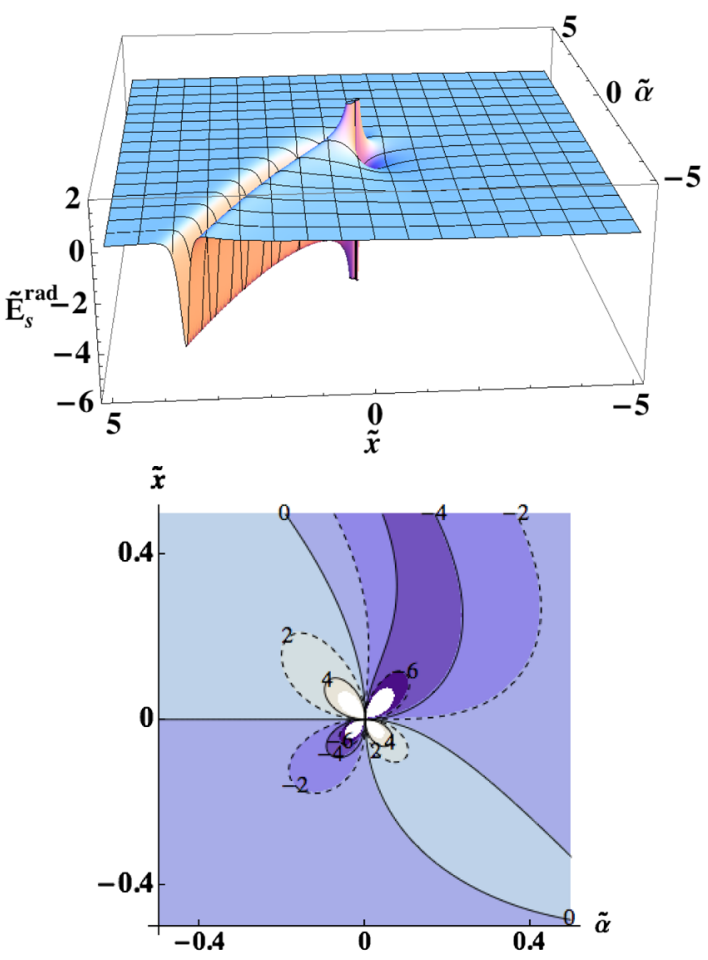

FIG. 7. Top: The longitudinal radiation wakefield $\tilde{E}_{s}^{\mathrm{rad}}(\tilde{\alpha}, \tilde{x})=$ $E_{s}^{\mathrm{rad}} /\left(e \gamma^{4} / R^{2}\right)$ of a single particle with $\gamma=100$. This figure is rotated for better visualization of the features in the wakefield. Bottom: The contour plot showing the cloverleaf pattern in the central part of $\tilde{E}_{s}^{\mathrm{rad}}(\tilde{\alpha}, \tilde{x})$.

$\beta^{2}=1$ while keeping the $\gamma$ dependence, as a universal equation for $\tilde{\psi}(\tilde{x}, \tilde{\alpha})$,

$$
\tilde{x}^{2}+2 \tilde{\alpha} \tilde{\psi}+(\tilde{x}-1) \tilde{\psi}^{2}-\tilde{\psi}^{4} / 12=0,
$$

where $\tilde{x} \equiv x \gamma^{2}, \tilde{\alpha} \equiv \alpha \gamma^{3}$, and $\tilde{\psi} \equiv \psi \gamma$. Dropping the $\alpha^{2}$ term is justified for most of the region in $(\alpha, x)$ space except for the vicinity of the negative $\alpha$ axis, where $\alpha^{2}$ may be comparable to or larger than $x^{2}$ and $2 \alpha \psi$. Since synchrotron radiation at the backward direction of the motion of a relativistic particle is weak, it is expected that the fields at this region are not important. Therefore the universal equation can be solved numerically once for $\tilde{\psi}$ and apply to a large range of beam parameters. It is worth discussing the amplitude scaling of $\left(\phi-\beta A_{s}\right)$ and $E_{s}$ with $\gamma$. From Eq. (17), $\left(\phi-\beta A_{s}\right)$ is proportional to $\left(1-\gamma^{-2} / 2\right) \psi^{-1}(1-\beta \sin \eta)^{-1}\left[1-\beta^{2} \cos (\alpha+\psi)\right]$ for its energy dependency. Since

$$
\begin{aligned}
& \psi^{-1}=\gamma \tilde{\psi}^{-1}, \quad \gamma \beta \cos \eta \approx \tilde{\psi} / 2-\tilde{x} / \tilde{\psi}, \\
& (1-\beta \sin \eta)^{-1} \approx \gamma^{2}(1+\beta \sin \eta) /\left[1+(\tilde{\psi} / 2-\tilde{x} / \tilde{\psi})^{2}\right], \\
& 1-\beta^{2} \cos (\alpha+\psi)=\gamma^{-2}\left[1-\frac{\beta^{2} \tilde{x}^{2} \gamma^{-2}-\tilde{\psi}^{2}}{2\left(1+\tilde{x} \gamma^{-2}\right)}\right] \approx \gamma^{-2},
\end{aligned}
$$

it is obvious that $\left(\phi-\beta A_{s}\right) \sim \gamma$ to the lowest order, therefore $E_{s}=-\partial\left(\phi-\beta A_{s}\right) / \partial \xi \sim \gamma^{3} \partial\left(\phi-\beta A_{s}\right) / \partial \tilde{\alpha} \sim \gamma^{4}$, as long as $(1+\beta \sin \eta)$ weakly depends on $\gamma$. The latter condition can be shown to be true for most of the region in the $(\tilde{\alpha}, \tilde{x})$ plane except for the vicinity of the negative $\tilde{\alpha}$ axis.

$\tilde{E}_{s}^{\mathrm{rad}} \equiv E_{s}^{\mathrm{rad}} /\left(e \gamma^{4} / R^{2}\right)$ is plotted as a function of $\tilde{\alpha}$ and $\tilde{x}$ in Fig. 7. It is interesting that, in addition to the slowly dropping off far field for $\tilde{\alpha} \gg 1$ similar to the result from 1D model, a pronounced feature appears in Fig. 7-a long trough extending from $(\tilde{\alpha}=0, \tilde{x}=0)$ to the corner of the figure. This trough corresponds to large negative values of $E_{s}^{\mathrm{rad}}$ and has a combination of the characteristics of the far field and the near field, i.e., its width in $\alpha$ is narrow but the length in $x$ is long. Furthermore, a cloverleaf pattern appears immediately surrounding the emitting particle, which is also shown in Fig. 7.

Next we discuss the long trough structure in Fig. 7. This negative spike is due to the $(1-\beta \sin \eta)^{3}$ factor in the denominator of $E_{s}^{\mathrm{rad}}$ when $\sin \eta=1$, while the field drops to 0 if $\sin \eta=\beta$. Therefore it is most interesting to investigate the behavior of $E_{s}^{\text {rad }}$ between $\beta \leq \sin \eta \leq 1$ or $-\gamma^{-1} \leq \cos \eta \leq \gamma^{-1}$. Noticing that $E_{s}^{\mathrm{rad}} /\left(e \beta^{3} / R^{2}\right)=$ $f_{1} \cdot f_{2}$, where $f_{1}=(\sin \eta-\beta) /(1-\beta \sin \eta)^{3}$ and $f_{2}=$ $\cos (\alpha+\psi+\eta) / \psi \approx-\left[2 x+x^{2}+(\psi / \beta)^{2}\right] /\left[2\left(\psi^{2} / \beta\right)\right]=$ $-\left(\lambda^{2} / \mu^{2}+1\right) / 2 \beta$. Here we only consider the region $x \geq 0$ and define $\lambda^{2}=2 x+x^{2}, \quad \mu=\psi / \beta=\nu+$ $\sqrt{\nu^{2}+\lambda^{2}}$, and $\nu=\cos \eta$. Both $f_{1}$ and $f_{2}$ are only functions of $\nu$ for fixed $x$ and $\beta$. It can be easily shown that $f_{1}(\nu)$ has a narrow spike as $f_{1}\left(-\gamma^{-1}\right)=f_{1}\left(\gamma^{-1}\right)=0$ and $f_{1}(0) \approx 4 \gamma^{4}$. On the contrary, $f_{2}(\nu)$ varies slowly when $\tilde{x} \gg 1$ or $\gamma^{2} \lambda^{2} \gg 1$, as $d f_{2} / d \nu>0$ and $\left[f_{2}\left(\gamma^{-1}\right)-\right.$ $\left.f_{2}\left(-\gamma^{-1}\right)\right] / f_{2}(0)=2 \sqrt{1+\gamma^{2} \lambda^{2}} /\left(\gamma^{2} \lambda^{2}\right) \approx 0$. Hence, the position of the spike for $x \geq 0$ can be approximated by setting $\eta=\pi / 2$ in Eqs. (2) and (3). The result (shown in Fig. 8) is

$$
\alpha=\cos ^{-1}[1 /(1+x)]-\beta \sqrt{2 x+x^{2}} .
$$

The above expression indicates that $\eta \neq \pi / 2$ when $x<0$, as can be seen from Fig. 1. Therefore the spike

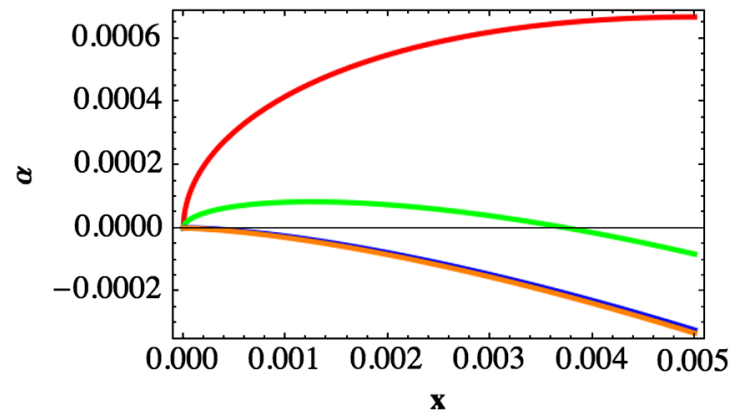

FIG. 8. Position of the negative spike in $E_{s}$ for $x>0$ and $\gamma=$ $10,20,100,1000$ (red, green, blue, orange curves, respectively) from Eq. (22). It is worth noting that the accuracy of the position is $O\left(\gamma^{-3}\right)$; therefore the curves for $\gamma=10,20$ are estimates of the peak position for the range shown. 
gradually disappears on the inner side of the particle's trajectory and $E_{s}^{\mathrm{rad}}$ becomes smooth.

To find the peak longitudinal electric field at the location specified by Eq. (22), one can use

$$
\psi=\beta \sqrt{2 x+x^{2}} \quad \cos (\alpha+\psi+\eta)=-\sqrt{1-(1+x)^{-2}}
$$

which is valid when $x>0$ (or $x<-2$ but this condition will never be fulfilled because $x \geq-1$ ). Therefore, the peak longitudinal radiation field at the trough is

$$
E_{s}^{\mathrm{rad}} \approx-\frac{e \beta^{2}}{R^{2}(1+x)(1-\beta)^{2}} \approx-\frac{4 e \beta^{2} \gamma^{4}}{R^{2}(1+x)} .
$$

Note that when $x \rightarrow 0$, this peak field approaches a value that is twice larger than $E_{s}^{\mathrm{rad}}\left(\alpha \rightarrow 0^{+}, x=0\right)$ in Eq. (19) indicating the discontinuous property of the single particle radiation wakefield at the origin. Since $x \ll 1$ for typical beam size, this peak field amplitude is almost constant across the beam, thus providing a strong radial coupling between particles over a long distance which is not present in the 1D models.

The angular width of the spike can be estimated by the node of $E_{s}^{\text {rad }}$, which corresponds to $\nu= \pm \gamma^{-1}$. Hence, $\Delta \alpha \approx \gamma^{-3}$ from Eqs. (2) and (3). This result is consistent with the width of the spiky feature in the 1D models. The radiation field from a single particle has a strong Fourier component at frequency $\sim \gamma^{3} \Omega_{c}$, where $\Omega_{c}=c / R$ is the angular frequency of the circular motion, confirming that the trough is produced by the radiation inside the synchrotron radiation cone.

\section{LONGITUDINAL CSR FIELD OF A BEAM}

In Eqs. (14) and (15), both $E_{s}^{\mathrm{rad}}$ and $E_{s}^{\mathrm{vel}}$ depend on bending radius $R$ explicitly through the factor $R^{-2}$ and implicitly through $\psi$ and $\eta$. Here we only consider the explicit dependence which demands $\sigma_{x} / R \ll 1$ such that to the lowest order $E_{s}^{\mathrm{rad}}$ and $E_{s}^{\mathrm{vel}}$ is independent of $R$, where $\sigma_{x}$ is the transverse spot size of the 2D Gaussian beam. Under this condition, we can calculate the longitudinal electric field for a beam with arbitrary shape through a convolution,

$\mathcal{E}_{s}^{i}(\xi, x)=\iint_{-\infty}^{+\infty} E_{s}^{i}\left(\xi-\xi^{\prime}, x-x^{\prime}\right) \rho\left(\xi^{\prime}, x^{\prime}\right) d \xi^{\prime} d x^{\prime}$,

where $i$ denotes either rad or vel.

\section{A. Steady-state CSR calculation}

Because of the strong spiking of $E_{s}^{\mathrm{rad}}\left(E_{s}^{\mathrm{vel}}\right)$, if the convolution is done numerically over the bunch shape, an angular resolution of at least $\Delta \alpha \sim 1 / \gamma^{3}$ (higher) is needed. For $\gamma$ on the order of $10^{3}$ or more, one has to resort to integration by parts or other techniques. Assuming the emitting particle has been in uniform circular motion with constant radius under the magnetic field forever, $\left(\phi-\beta A_{s}\right)$ is only a function of $\xi$ and $x$. It is straightforward to show that the tangential electric field of a beam (including both velocity and radiation fields) is

$$
\begin{aligned}
\mathcal{E}_{s}(\xi, x)= & -\left.\iint_{-\infty}^{+\infty} \frac{\left(\phi-\beta A_{s}\right)}{\partial \xi}\right|_{\xi-\xi^{\prime}, x-x^{\prime}} \\
& \times \rho\left(\xi^{\prime}, x^{\prime}\right) d \xi^{\prime} d x^{\prime} \\
= & -\left.\iint_{-\infty}^{+\infty}\left(\phi-\beta A_{s}\right)\right|_{\xi-\xi^{\prime}, x-x^{\prime}} \\
& \times \frac{\rho\left(\xi^{\prime}, x^{\prime}\right)}{\partial \xi^{\prime}} d \xi^{\prime} d x^{\prime} .
\end{aligned}
$$

Equation (25) includes both the velocity field and radiation field, and it is dominated by the radiation field $\mathcal{E}_{s}^{\mathrm{rad}}$ at high beam energy. However, at low beam energy we expect the opposite. As the well-known parabolic cylinder function result in the 1D model relies on the single particle farfield result for $x=0$ and $\beta=1$, we expect deviations from this result will occur for the field off the central trajectory in the 2D model with low energy and large spot sizes. Although one would think that the velocity field will lead to a diverging result as soon as a finite energy beam is used, which is the rationale of the so-called "renormalization" procedure [6], it turns out the result is converging as $\Delta \xi$ is decreased. This shows that the velocity field for a beam in a 2D model is actually finite. In the Appendix, the convergence is proved for the space charge (velocity) field of a Gaussian beam in a uniform straight-line motion, which gives insight on the difference of the convergence properties in $1 \mathrm{D}$ and $2 \mathrm{D}$ models. However, to prove the convergence for the case of circular motion is difficult and in practice we need to monitor the convergence of result.

Figure 9 compares $\mathcal{E}_{s}^{\text {rad }}$ from Eq. (23) and $\mathcal{E}_{s}$ calculated from Eq. (25) for a 2D Gaussian beam with $\sigma_{x}=\sigma_{s}=$ $200 \mu \mathrm{m}, R=100 \mathrm{~cm}$, and $\gamma=10,100$. Note that the convolution in Eqs. (23) and (25) can be done using electric field or potential kernel defined in the scaled spatial variables $\tilde{\alpha}, \tilde{x}$. Similarly, one can define the scaled beam size $\tilde{\sigma}_{s}=\sigma_{s} \gamma^{3} / R$ and $\tilde{\sigma}_{x}=\sigma_{x} \gamma^{2} / R$ to be used in Eqs. (23) and (25). With this change of variables, it is easy to understand the qualitative behavior of the longitudinal CSR field. For example, Fig. 9 shows that $\mathcal{E}_{s}^{\text {rad }}$ resembles the cloverleaf pattern in the central part of the single particle wakefield, which is a consequence of the scaled beam size $\tilde{\sigma}_{s}$ and $\tilde{\sigma}_{x}$ being smaller or close to the size of the single particle cloverleaf pattern in Fig. 7. Figure 9 also shows that $\mathcal{E}_{s}$ is dominated by the velocity field at $\gamma=10$; while for $\gamma=100$, both $\mathcal{E}_{s}$ and $\mathcal{E}_{s}^{\text {rad }}$ approach the parabolic cylinder solution of the far-field response in the 1D model since the velocity field scales as $\gamma^{-2}$ and $\tilde{\sigma}_{s}$ is much larger than $\tilde{\sigma}_{x}$ at this energy, making the scaled beam shape more 1D-like. Figure 10 shows that the agreement of the on-axis longitudinal field with the parabolic cylinder solution improves quickly for higher beam energy. However, deviation from this solution is still observed in Fig. 10 across the $x$ 

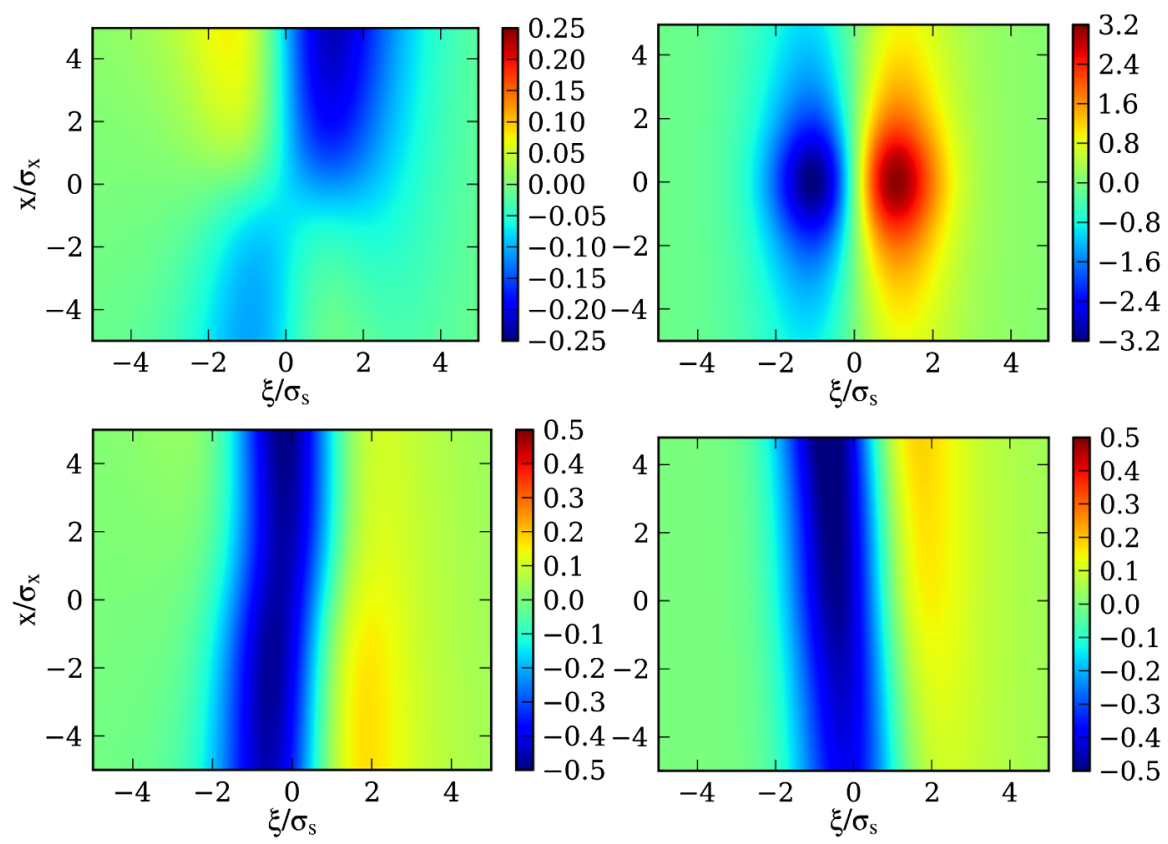

FIG. 9. Top row: The longitudinal radiation field $\mathcal{E}_{s}^{\text {rad }}$ (left) and the total longitudinal field $\mathcal{E}_{s}$ (right) for a $2 \mathrm{D}$ Gaussian beam of $\sigma_{x}=\sigma_{s}=200 \mu \mathrm{m}, \gamma=10$, beam charge $Q=1 \mathrm{nC}$ and $R=100 \mathrm{~cm}$. Bottom row: $\mathcal{E}_{s}^{\text {rad }}$ (left) and the total longitudinal field $\mathcal{E}_{s}$ (right) for the same parameters except for $\gamma=100$. All fields are in units of $\mathrm{MV} / \mathrm{m}$.

dimension which is a result of the asymmetry in the single particle wakefield in Fig. 7. For beams with large aspect radio $\sigma_{x} / \sigma_{s}$, this variation along the $x$ direction is more pronounced.

\section{B. Transient CSR calculation}

The method to calculate the steady-state field can be used for the transient CSR field as long as the longitudinal beam size is sufficiently small. The single particle wakefield $E_{s}^{i}$ in Eq. (23) and potential $\left(\phi-\beta A_{s}\right)$ in Eq. (25) will be replaced by the corresponding transient wakefield

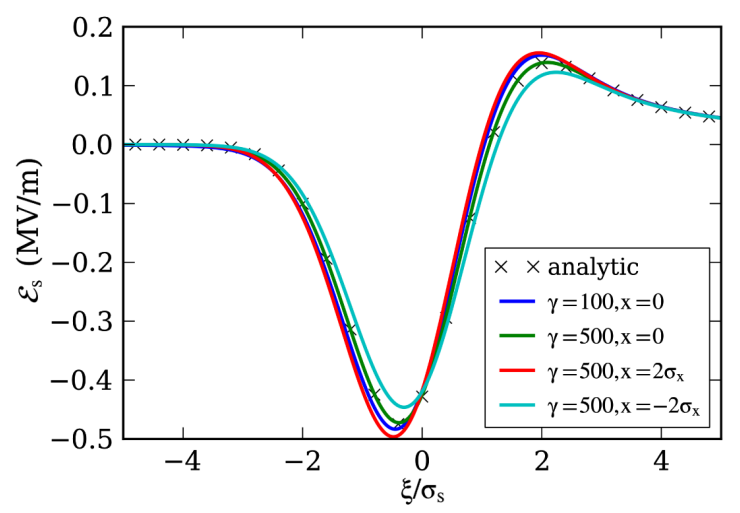

FIG. 10. The on-axis longitudinal CSR field $\mathcal{E}_{s}$ for the same parameters in Fig. 9 and with $\gamma=100$, 500, which corresponds to scaled longitudinal beam spot size of 200, 25000 , respectively. For $\gamma=500$, the fields at $x= \pm 2 \sigma_{x}$, i.e., $\tilde{x}= \pm 100$ are also shown. The analytic result from 1D models [5,6] is plotted for comparison. and potential. The simplest case when a beam enters a uniform magnetic field is considered here as a demonstration, which is also of practical interest for benchmarking self-consistent simulation. Before the beam enters the magnetic field region, it moves along a straight path with constant velocity. For a single particle, this means that the field and potential is determined by Eqs. (14) and (17) when $\psi(\alpha, x) \leq \psi_{0}=s / R$, where $s$ is the distance the particle travels into the bend. While for $\psi(\alpha, x)>\psi_{0}$, the contribution is from the motion in the straight section of the trajectory. It can be easily shown that in this case, $E_{s}^{\mathrm{rad}}=0$ and

$\left(\phi-\beta A_{s}\right)=\frac{e \beta}{R} \frac{1-\beta^{2} \cos \alpha^{\prime}}{l / R+\psi_{0}-\beta^{2}\left[1-(1+x) \cos \alpha^{\prime}\right]}$,

where $\alpha^{\prime}=\alpha+\psi_{0} . l / R$ is the normalized distance between the retarded position of the particle and the entrance of the magnet, which can be solved in the following equation:

$$
\frac{l / R+\psi_{0}}{\sqrt{\left[(1+x) \sin \alpha^{\prime}+l / R\right]^{2}+\left[1-(1+x) \cos \alpha^{\prime}\right]^{2}}}=\beta .
$$

For a beam with a finite longitudinal length $L$, each particle enters the magnet at a different time. Therefore the location of the boundary $\psi=\psi_{0}$ in the $(\alpha, x)$ plane varies according to the initial longitudinal position of the particle in the beam, causing a slightly different field near this boundary for each particle. The $\Delta \psi_{0}$ between the first and last particle is $L / R$. An estimate of the width 

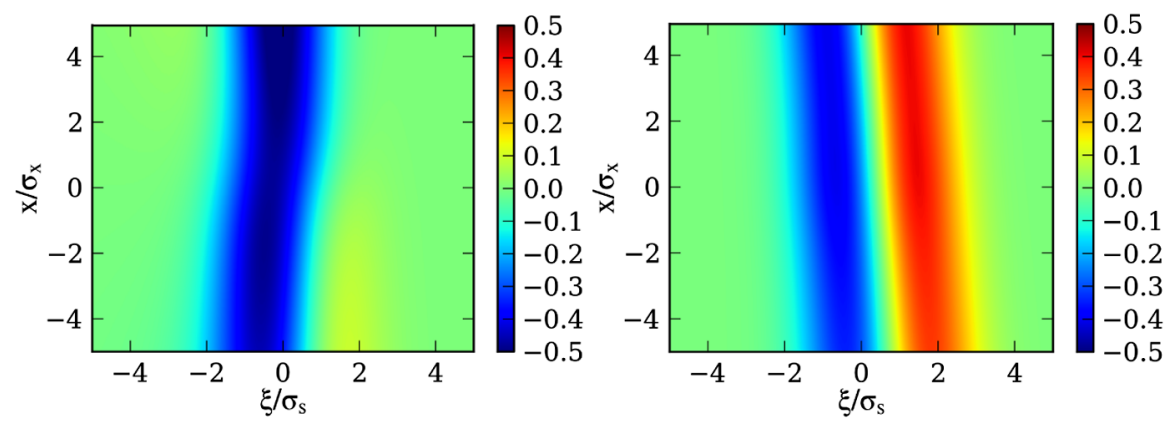

FIG. 11. The transient longitudinal radiation field $\mathcal{E}_{s}^{\mathrm{rad}}$ (left) and the transient total longitudinal field $\mathcal{E}_{s}$ (right) (in units of MV/m) when beam has rotated $10^{\circ}$. The beam parameters are the same as in Fig. 9 and $\gamma=100$.

in $\alpha$ of the boundary region is $\Delta \alpha=2^{-1} \cdot 3^{2 / 3} \psi_{0}^{2} \Delta \psi_{0}$. Furthermore, the potential from Eq. (26) is typically much smaller than the potential from Eq. (25). Therefore, when the boundary region width is sufficiently small compared to the beam length in $\alpha$, i.e. $\Delta \alpha /(L / R) \ll 1$, one can adopt Eqs. (23) and (25) for the transient CSR calculation. As the CSR field within the beam will reach steady state when $\psi_{0}>\psi_{0}^{\max }=(24 L / R)^{1 / 3}$, for a transient calculation, the ratio $\Delta \alpha /(L / R)<2^{-1} \cdot 3^{2 / 3}\left(\psi_{0}^{\max }\right)^{2}=$ $6 \cdot 3^{1 / 3}(L / R)^{2 / 3}$. For typical beam size, the condition $\Delta \alpha /(L / R) \ll 1$ is well satisfied.

Figures 11 and 12 show the transient longitudinal CSR fields when the beam has rotated $10^{\circ}$ in a bend for same beam parameters used in Fig. 9. The transient fields are substantially different than the 1D analytic result from Eq. (88) in Ref. [6]. For example, although both the 1D and 2D on-axis results approach the steady-state parabolic cylinder function solution as $s$ increases, the behavior predicted by the transient term in the 1D model, which is responsible for the peak around $\xi / \sigma_{s}=4$ in Fig. 12, is generally not seen in the $2 \mathrm{D}$ results.

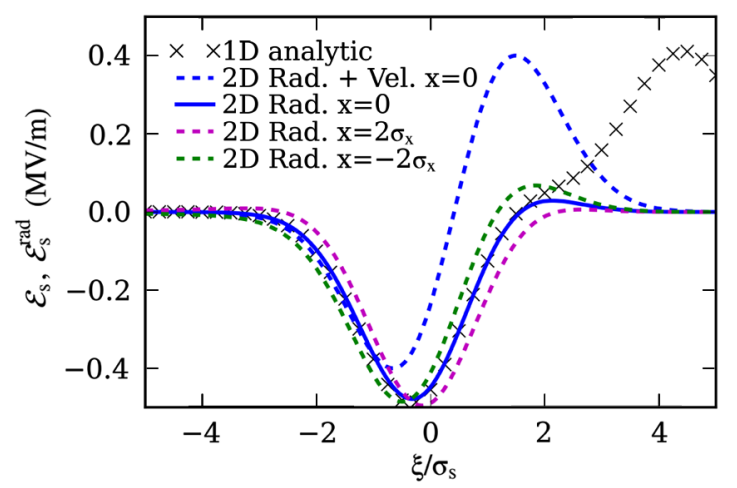

FIG. 12. Comparison of the transient longitudinal CSR field $\mathcal{E}_{s}$ (blue dashed curve) and $\mathcal{E}_{s}^{\text {rad }}$ (blue solid curve) with the 1D analytic result from Eq. (88) of Ref. [6] for the same beam parameters in Fig. 11. The 1D result applies to ultrarelativistic beams. The off-axis field at $x= \pm 2 \sigma_{x}$ shows the transverse variation of $\mathcal{E}_{s}^{\text {rad }}$ in $2 \mathrm{D}$.

\section{Coordinate transform}

In Sec. IV, we calculate the CSR field for a 2D beam assuming a local Gaussian beam density profile in the accelerator (Frenet-Serret) coordinates. However, in beam dynamics simulations, we usually initialize the particle beam in Cartesian coordinates outside of the uniform magnetic field region and then propagate the beam selfconsistently into this region. The edge of the magnetic field region is assumed to be perpendicular to the beam's entrance velocity and there is no initial velocity spread for the beam particles. To benchmark such a simulation with the numerical model in this paper, we can first avoid the effect of the CSR field on the particle by using a low charge beam. With this simulation setup, each beam particle will bend in the magnetic field region with the same bending radius but around different origins. This is illustrated in Fig. 13. The density profile of the beam thus changes as it propagates, which will lead to a deviation of the CSR field from those calculated using the initial Gaussian profile. For careful benchmarking between the simulation and the 2D numerical model, this needs to be taken into account when the bending angle is large. A more general study of the coordinate transform is discussed in Ref. [13]; here we focus on the transform for the specific simulation setup illustrated in Fig. 13.

From Fig. 13, we can see that particles on a vertical line initially remain on a vertical line at any instance during the circular motion; therefore a particle at point $A$ with accelerator coordinates $(\xi \equiv \alpha R, x)$ can be mapped back to its Cartesian coordinate $\left(\xi_{0} \equiv \widehat{P A^{\prime \prime}}, x_{0} \equiv \overline{A A^{\prime \prime}}\right)$ before entering the magnetic bend using the following relations:

$$
\begin{gathered}
\omega=\cos ^{-1}[(1+x / R) \sin (\alpha+\theta)], \\
\xi_{0}=R\left(\frac{\pi}{2}-\theta-\omega\right), \\
x_{0}=(R+x) \cos (\alpha+\theta)-\sqrt{R^{2}-(R+x)^{2} \sin ^{2}(\alpha+\theta)} .
\end{gathered}
$$




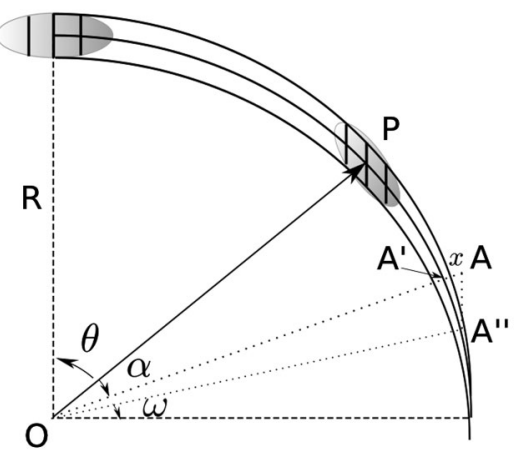

FIG. 13. Trajectories (solid curves) of three beam particles which are initially at the center of the beam and separated by equal distance vertically. Their trajectories are arcs with the same curvature but shifted in the vertical direction by the initial separation distance. Particles on a vertical line remain on a vertical line as shown but their relative position to the center of the beam $P$ has changed. The center of the beam has rotated by an angle $\theta$ and a particle at point $A$ nearby can be described by the accelerator coordinates $(\alpha, x)$ or $(\xi \equiv \alpha R, x)$. Before entering the magnetic bend, the Cartesian coordinates of this particle relative to the beam center is $\left(\xi_{0} \equiv \widehat{P A^{\prime \prime}}, x_{0} \equiv \overline{A A^{\prime \prime}}\right) . A^{\prime}, A^{\prime \prime}$ are the intersections of $P$ 's trajectory with $\overline{O A}$ and with $\overline{A A^{\prime \prime}}$, respectively, where $\overline{A A^{\prime \prime}}$ is a vertical line.

The Jacobian of this transform is

$$
J=\left[\begin{array}{ll}
\frac{\partial \xi_{0}}{\partial \xi} & \frac{\partial \xi_{0}}{\partial x} \\
\frac{\partial x_{0}}{\partial \xi} & \frac{\partial x_{0}}{\partial x}
\end{array}\right],
$$

where

$$
\begin{aligned}
& \frac{\partial \xi_{0}}{\partial \xi}=\kappa \cos (\alpha+\theta), \quad \frac{\partial \xi_{0}}{\partial x}=\frac{R}{R+x} \kappa \sin (\alpha+\theta), \\
& \frac{\partial x_{0}}{\partial \xi}=\frac{(R+x) \sin (\alpha+\theta)}{R}(\kappa-1), \\
& \frac{\partial x_{0}}{\partial x}=\cos (\alpha+\theta)+\kappa \sin ^{2}(\alpha+\theta),
\end{aligned}
$$

and $\kappa=\left[R^{2} /(R+x)^{2}-\sin ^{2}(\alpha+\theta)\right]^{-1 / 2}$. So the beam density transforms as

$$
\rho(\xi, x ; \theta)=\rho_{0}\left(\xi_{0}, x_{0} ; 0\right)|J| .
$$

Figure 14 shows the coordinate transform $(\xi, x) \rightarrow$ $\left(\xi_{0}, x_{0}\right)$ of the particle for the parameters in Fig. 9 when the beam has rotated $\theta=10^{\circ}=0.175 \mathrm{rad}$. The dominant effect introduced by the transform is a shear in $\xi_{0}(\xi, x)$ shown in the top panel of Fig. 14. Figure 15 shows the beam density in the Cartesian coordinate $\rho(Z, X)$ which is mapped from the density in the accelerator coordinate $\rho(\xi, x ; \theta)$ by $Z=(R+x) \sin (\theta+\xi / R)-R \sin \theta$ and $X=$ $(R+x) \cos (\theta+\xi / R)-R \cos \theta$ for better comparison with simulation in the Cartesian geometry. The beam
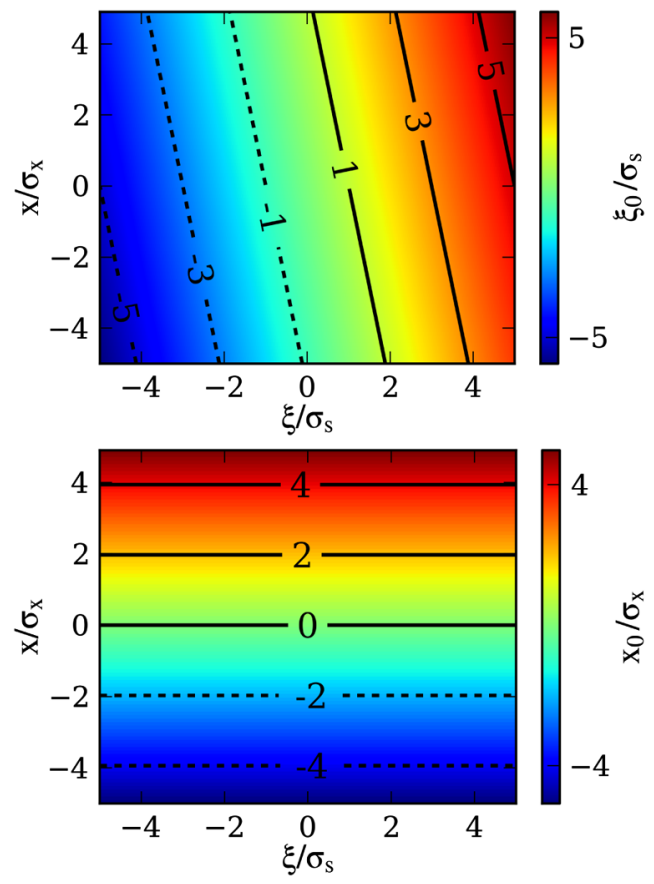

FIG. 14. $(\xi, x) \rightarrow\left(\xi_{0}, x_{0}\right)$ transform for the parameters in Fig. 9, i.e., $\sigma_{x}=\sigma_{s}=200 \mu \mathrm{m}, \gamma=100$, and $R=100 \mathrm{~cm}$, when the beam has rotated $\theta=10^{\circ} \approx 0.175 \mathrm{rad}$. The major effect of the transform is seen in the plot of $\xi_{0}(\xi, x)$ where $\xi_{0}$ has a $x$-dependent shift to $\xi$.
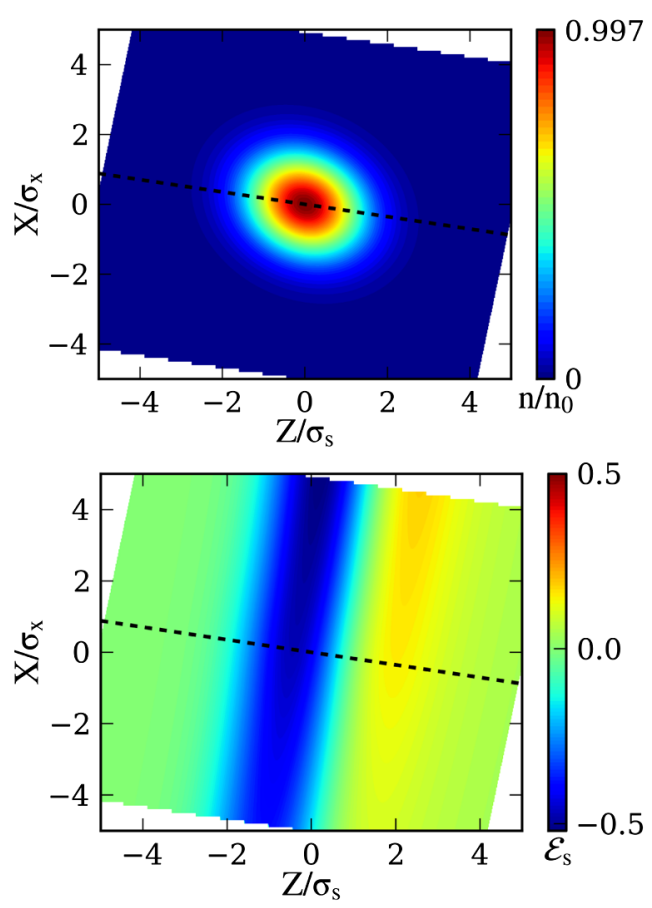

FIG. 15. The beam density $n / n_{0}$ and the total longitudinal CSR field (in $\mathrm{MV} / \mathrm{m}$ ) in the Cartesian coordinates for a bending angle of $\theta=0.175 \mathrm{rad}$ and the same parameters in Fig. 14. $n_{0}$ is the peak beam density before it enters the magnetic field region. 
is tilted in the Cartesian geometry because of both the coordinate transform defined in Eq. (30) and the mapping into the Cartesian geometry. The former leads to a tilt of the semiaxes of the Gaussian shape beam with respect to the tangent of the beam trajectory at a bending angle of $\theta=10^{\circ}=0.175 \mathrm{rad}$ (denoted by the dashed line in Fig. 15), while the latter results in a overall rotation of angle $\theta$. Compared to the result in Fig. 9, the total longitudinal CSR field is also seen to be tilted.

\section{SUMMARY}

We have presented a simple model for CSR calculation for a beam with a predefined trajectory using the Liénard-Wiechert formula. This model is an extension of the well-known 1D line charge model to two spatial dimensions in the bending plane. The use of a $2 \mathrm{D}$ model is motivated by the need to benchmark PIC simulation results and the demonstration in the Appendix that a line charge model leads to diverging space charge potential and fields for a coasting beam. In the 1D line charge model, the longitudinal electric field from a single particle in the steady-state is independent of the angular position of the particle and is only a function of the relative angular position of the observation point. This allows a convenient convolution procedure to replace the summation process for the CSR calculation of a smooth beam profile. In our model, we apply a similar convolution algorithm in $2 \mathrm{D}$ by assuming the single particle wakefield kernel is invariant if the source particle is rotated around the origin or displaced slightly in the radial direction by an amount comparable to the beam size, which can be justified when the beam size is much smaller than the bending radius. For the longitudinal radiation field of a beam, Eq. (23) can be used. We note that the diverging velocity field does not require renormalization in the 2D model; therefore the total CSR field including both the velocity field and the radiation field can also be calculated from Eq. (23). Nonetheless numerical convergence needs to be monitored and imposes a severe limitation for the integration of a kernel with such strong singularity. In this paper, the convergence is significantly improved by using integration by parts, i.e., Eq. (25), however it may be further improved by other analytic/numerical integration methods such as singularity subtraction/cancellation, coordinate transform, and the integrated Green's function technique [14].

The properties of the single particle wakefield kernel in the bending plane are investigated in detail. This kernel is calculated using the exact Liénard-Wiechert formula Eq. (14) for the longitudinal radiation field and Eq. (17) for the wake potential. Approximation is only made in the evaluation of the retarded angle for which a new equation (10) sufficiently accurate for the parameters of interest is derived from the exact equation (2). The result from Eq. (10) is compared to the exact solution in 2D and good agreement is found. Simplified solutions from this equation are also given in Eqs. (11)-(13) and compared to the commonly used approximation Eqs. (4) and (7) in 1D models, revealing the validity range of the $1 \mathrm{D}$ result. A universal equation (21) for the retarded angle can be further derived from Eq. (10). Using Eqs. (14) and (21), the major features of the single particle longitudinal wakefield can be elucidated. Similar to the 1D result, this kernel exhibits a farfield pattern for angular distance $\alpha \gg \gamma^{-3}$, a spiky nearfield pattern for $\alpha \leqslant \gamma^{-3}$ which is the consequence of the forward synchrotron radiation cone of the radiation from a relativistic particle. In $2 \mathrm{D}$, this near-field pattern extends to a large distance independent of beam energy in the outer radial direction but quickly drops off in the inner direction, which is again the result from the synchrotron radiation cone. This finding may have important consequence when the beam is modulated or a material boundary is present in the radial direction. With the help of the universal equation, one can define scaled beam sizes in the longitudinal and radial directions, i.e., $\tilde{\sigma}_{s}=$ $\sigma_{s} \gamma^{3} / R$ and $\tilde{\sigma}_{x}=\sigma_{x} \gamma^{2} / R$. Thus, the beam CSR field can be calculated by a convolution of the scaled beam density profile with the universal beam wakefield kernel. The results from our model indicate that the well-known parabolic cylinder functions is essentially the near-axis far-field result when $\tilde{\sigma}_{s} \gg 1$ and $\tilde{\sigma}_{x} / \tilde{\sigma}_{s} \ll 1$. While for beams with longitudinal size or modulation on the order of $R / \gamma^{3}$, or with an aspect ratio $\tilde{\sigma}_{x} / \tilde{\sigma}_{s}$ approaching or larger than unity, large deviation from the parabolic cylinder result is expected.

Finally, our model should be considered as an extension of the core algorithm of the 1D model to 2D. Therefore transient effects and parallel-plate shielding can be included in the same fashion as in 1D models. For example, it is shown that the transient effect in 2D can be calculated and it differs substantially from the 1D result due to the spreading of radiation in $2 \mathrm{D}$. Hence, the $2 \mathrm{D}$ model will be useful for benchmarking the CSR field in a multidimensional PIC simulation, for which self-consistent modeling is possible. In a future work, we will explore parallel-plate shielding through the method of image charge and possible 3D effects.

\section{ACKNOWLEDGMENTS}

This work is performed under the auspices of DOE by the Los Alamos National Security/Los Alamos National Laboratory, and supported by Laboratory Directed Research and Development program. We acknowledge useful discussions with Robert Ryne, Nikolai Yampolsky, Quinn Marksteiner, Kip Bishofberger, Cliff Fortgang, Leanne Duffy, Peter Walstrom, and Henry Freund and comments from Hui-chun Wu. 


\section{APPENDIX: SPACE CHARGE FIELD IN 1D AND 2D MODELS}

Space charge field is the only field for a beam coasting at a constant velocity. The calculation here can be useful to illustrate the effectiveness of the numerical methods to evaluate those fields with singularity at the position of the source particle. We consider the space charge field for a 2D Gaussian beam moving in the $+x$ direction. The beam lies in the $x-y$ plane and is infinitesimally thin in the $z$ direction, therefore its charge distribution is only "2D". (We need to distinguish this from the situation in a 2D PIC simulation where the beam actually has infinite length in $z$. In fact, the proper simulation to compare to is a $3 \mathrm{D}$ one with a beam of one cell thick in $z$.) Such a 2D beam is more realistic than the line charge distribution in a 1D model, but its field is less difficult to solve than a 3D distribution. We will show that the line charge model leads to diverging fields and potentials in the space charge field.
In the beam's rest frame, the electrostatic potential $\phi$ can be calculated using the Green's function of the Poisson's equation,

$\phi(x, y, z)=\iiint_{-\infty}^{+\infty} \frac{\rho\left(x^{\prime}, y^{\prime}, z^{\prime}\right) d x^{\prime} d y^{\prime} d z^{\prime}}{\left[\left(x-x^{\prime}\right)^{2}+\left(y-y^{\prime}\right)^{2}+\left(z-z^{\prime}\right)^{2}\right]^{1 / 2}}$.

The integrand in this integral is singular for a position inside the beam where the density is nonzero. However, by using a numerical technique which expresses $1 /\left[\left(x-x^{\prime}\right)^{2}+\left(y-y^{\prime}\right)^{2}+\left(z-z^{\prime}\right)^{2}\right]^{1 / 2}=1 / R$ as the integral of a Gaussian function, i.e.,

$$
\frac{1}{R}=\frac{1}{|R|}=\sqrt{\frac{2}{\pi}} \int_{0}^{\infty} e^{-\lambda^{2} R^{2} / 2} d \lambda,
$$

the Kheifets-Bassetti-Erskine [15] formula for a Gaussian beam $\rho(x, y, z)=\frac{Q}{(2 \pi)^{3 / 2} \sigma_{x} \sigma_{y} \sigma_{z}} e^{-\left(x^{2} / 2 \sigma_{x}^{2}\right)} e^{-\left(y^{2} / 2 \sigma_{y}^{2}\right)} e^{-\left(z^{2} / 2 \sigma_{z}^{2}\right)}$ can be derived:

$$
\phi(x, y, z)=\sqrt{\frac{2}{\pi}} \frac{Q}{\sigma_{x} \sigma_{y} \sigma_{z}} \int_{0}^{\infty} \frac{e^{-\left[x^{2} \lambda^{2} / 2\left(\lambda^{2} \sigma_{x}^{2}+1\right)\right]-\left[y^{2} \lambda^{2} / 2\left(\lambda^{2} \sigma_{y}^{2}+1\right)\right]-\left[z^{2} \lambda^{2} / 2\left(\lambda^{2} \sigma_{z}^{2}+1\right)\right]}}{\sqrt{\left(\lambda^{2}+\sigma_{x}^{-2}\right)\left(\lambda^{2}+\sigma_{y}^{-2}\right)\left(\lambda^{2}+\sigma_{z}^{-2}\right)}} d \lambda .
$$

Taking the limit $\sigma_{z} \rightarrow 0$, the potential of a $2 \mathrm{D}$ beam $\rho(x, y, z)=\frac{Q}{2 \pi \sigma_{x} \sigma_{y}} e^{-\left(x^{2} / 2 \sigma_{x}^{2}\right)} e^{-\left(y^{2} / 2 \sigma_{y}^{2}\right)} \delta(z)$ is

$$
\phi(x, y, z)=\sqrt{\frac{2}{\pi}} \frac{Q}{\sigma_{x} \sigma_{y}} \int_{0}^{\infty} \frac{e^{-\left[x^{2} \lambda^{2} / 2\left(\lambda^{2} \sigma_{x}^{2}+1\right)\right]-\left[y^{2} \lambda^{2} / 2\left(\lambda^{2} \sigma_{y}^{2}+1\right)\right]-\left(z^{2} \lambda^{2} / 2\right)}}{\sqrt{\left(\lambda^{2}+\sigma_{x}^{-2}\right)\left(\lambda^{2}+\sigma_{y}^{-2}\right)}} d \lambda
$$

Then the potential in the lab frame can be obtained after a Lorentz transformation. Since the Lorentz transformation does not introduce singularity in the calculation, one only needs to check the convergence of the result in the rest frame. The integral in Eq. (A3) does not involve a singular integrand and can be performed to verify that the result is finite in a $2 \mathrm{D}$ model. For the potential in the $2 \mathrm{D}$ plane at $z=0$, the integral in Eq. (A3) can be rewritten by variable change $\lambda \sigma_{x} \rightarrow \lambda$ as

$$
\phi(x, y, z=0)=\sqrt{\frac{2}{\pi}} \frac{Q}{\sigma_{y}} \int_{0}^{\infty} G_{2}(\lambda) d \lambda,
$$

where

$$
G_{2}(\lambda)=\frac{e^{-\left[\left(x / \sigma_{x}\right)^{2} \lambda^{2} / 2\left(\lambda^{2}+1\right)\right]}}{\sqrt{\lambda^{2}+1}} \frac{e^{-\left[\left(y / \sigma_{x}\right)^{2} \lambda^{2} / 2\left(\lambda^{2} \sigma_{y}^{2} \sigma_{x}^{-2}+1\right)\right]}}{\sqrt{\lambda^{2}+\sigma_{x}^{2} \sigma_{y}^{-2}}} .
$$

Defining

$$
G_{3}(\lambda)=\frac{1}{\sqrt{\left(\lambda^{2}+1\right)\left(\lambda^{2}+\sigma_{x}^{2} \sigma_{y}^{-2}\right)}},
$$

it is straightforward to show that $G_{2}$ has lower and upper bounds,

$$
e^{-(1 / 2)\left[\left(x / \sigma_{x}\right)^{2}+\left(y / \sigma_{y}\right)^{2}\right]} G_{3}(\lambda) \leq G_{2}(\lambda) \leq G_{3}(\lambda) .
$$

If we integrate Eq. (A5) over $\lambda$, we will obtain the lower bound and the upper bound of $\phi$ too. It turns out that

$$
\int_{0}^{\infty} G_{3}(\lambda) d \lambda=\frac{\sigma_{y}}{\sigma_{x}} K\left(1-\frac{\sigma_{y}^{2}}{\sigma_{x}^{2}}\right)
$$

where $K$ is the complete elliptic integral of the first kind, therefore

$$
e^{-(1 / 2)\left[\left(x / \sigma_{x}\right)^{2}+\left(y / \sigma_{y}\right)^{2}\right]} H\left(\sigma_{x}, \sigma_{y}\right) \leq \sqrt{\frac{\pi}{2}} \frac{1}{Q} \phi \leq H\left(\sigma_{x}, \sigma_{y}\right),
$$

where both the lower and the upper bound is proportional to $H\left(\sigma_{x}, \sigma_{y}\right)=K\left(1-\sigma_{y}^{2} / \sigma_{x}^{2}\right) / \sigma_{x}$, which is finite for finite $\sigma_{x}$ and $\sigma_{y}$. However, when $\sigma_{y}=0$, i.e., in the line charge case, $H\left(\sigma_{x}, 0\right)=\infty$, so $\phi(x, y=0) \rightarrow \infty$. 
A similar conclusion can be drawn for $\phi(x=0, y)$ if we swap $\sigma_{x}$ and $\sigma_{y}$ in the above calculation and let $\sigma_{x}=0$. One can also directly verify the potential in 1D line charge model diverges by bounding the following integral:

$$
\begin{aligned}
\phi(x, y=0, z=0) & =\sqrt{\frac{2}{\pi}} \frac{Q}{\sigma_{x}} \int_{0}^{\infty} \frac{e^{-\left[\left(x / \sigma_{x}\right)^{2} \lambda^{2} / 2\left(\lambda^{2}+1\right)\right]}}{\sqrt{\lambda^{2}+1}} d \lambda \\
& =\sqrt{\frac{2}{\pi} \frac{Q}{\sigma_{x}} \int_{0}^{\infty} G_{1}(\lambda) d \lambda} \\
\frac{e^{-\left(x / \sigma_{x}\right)^{2} / 2}}{\sqrt{\lambda^{2}+1}} & \leq G_{1}(\lambda) \leq \frac{1}{\sqrt{\lambda^{2}+1}}
\end{aligned}
$$

The lower and upper bounds of $\phi$ are both proportional to $\int_{0}^{\infty} 1 / \sqrt{\lambda^{2}+1} d \lambda=\left.\sinh ^{-1}(\lambda)\right|_{0} ^{\infty}=\infty$; therefore the result is infinite.

One can also show the electric fields for a Gaussian beam are infinite in the 1D line charge model and finite in the 2D model by taking the derivative of Eq. (A4). Although we only show the convergence of the result in the same plane of the 2D beam above, the convergence for the fields off the plane can be calculated in a similar manner. Therefore, the space charge field calculation can be included in a 2D model for CSR as long as the numeric calculation converges.
[1] R. Li, Phys. Rev. ST Accel. Beams 11, 024401 (2008).

[2] G. Bassi, J. Ellison, K. Heinemann, and R. Warnock, Phys. Rev. ST Accel. Beams 12, 080704 (2009).

[3] D. Gillingham and T. Antonsen, Phys. Rev. ST Accel. Beams 10, 054402 (2007).

[4] A. Novokhatski, Phys. Rev. ST Accel. Beams 14, 060707 (2011).

[5] J. Murphy, S. Krinsky, and R. Gluckstern, Part. Accel. 57, 9 (1997) [http://cds.cern.ch/record/1120287].

[6] E. Saldin, E. Schneidmiller, and M. Yurkov, Nucl. Instrum. Methods Phys. Res., Sect. A 398, 373 (1997).

[7] C. Mayes and G. Hoffstaetter, Phys. Rev. ST Accel. Beams 12, 024401 (2009).

[8] D. Sagan, G. Hoffstaetter, C. Mayes, and U. Sae-Ueng, Phys. Rev. ST Accel. Beams 12, 040703 (2009).

[9] R. D. Ryne, B. Carlsten, J. Qiang, and N. Yampolsky, arXiv: 1202.2409 .

[10] G. N. Afanasiev, V. M. Shilov, and V.P. Permiakov, Phys. Scr. 65, 145 (2002).

[11] G. A. Scott, Electromagnetic Radiation (Cambridge University Press, Cambridge, 1912).

[12] P. Goldreich and D. A. Keeley, Astrophys. J. 170, 463 (1971).

[13] G. Bassi, J. Ellison, K. Heinemann, and R. Warnock, Phys. Rev. ST Accel. Beams 13, 104403 (2010).

[14] R. D. Ryne, in Proceedings of IPAC12, New Orleans, LA, USA (2012), MOPPC085 [http://accelconf.web.cern.ch/ accelconf/IPAC2012/papers/moppc085.pdf], and reference therein.

[15] M. Bassetti and G. Erskine, Technical Report No. CERNISR-TH/80-06, 1980. 